\title{
Fluctuations in the open time of synaptic channels: an application to noise analysis based on charge
}

\author{
Hinnerk Feldwisch-Drentrup ${ }^{1}$, Adam B. Barrett ${ }^{2}$, Michael T. Smith, Mark C.W. van \\ Rossum* \\ Institute for Adaptive and Neural Computation \\ School of Informatics \\ University of Edinburgh \\ 10 Crichton Street \\ Edinburgh, EH8 $9 A B U K$
}

\begin{abstract}
Synaptic channels are stochastic devices. Even recording from large ensembles of channels, the fluctuations, described by Markov transition matrices, can be used to extract single channel properties. Here we study fluctuations in the open time of channels, which is proportional to the charge flowing through the channel. We use the results to implement a novel type of noise analysis that uses the charge rather than the current to extract fundamental channel parameters. We show in simulations that this charge based noise analysis is more robust if the synapse is located on the dendrites and thus subject to cable filtering. However, we also demonstrate that when multiple synapses are distributed on the dendrites, noise analysis breaks down. We finally discuss applications of our results to other biological processes.
\end{abstract}

Keywords: synaptic transmission, noise analysis, stochastic channels, neural variability

\footnotetext{
${ }^{*}$ Corresponding author

Email address: mvanross@inf.ed.ac.uk (Mark C.W. van Rossum)

${ }^{1}$ Currently at Bernstein Center Freiburg, University of Freiburg, Hansastr. 9a, 79104 Freiburg, Germany.

${ }^{2}$ Currently at School of Informatics, University of Sussex, Brighton BN1 9QJ, UK.
} 


\section{Introduction}

Neural activity and neural transmission rely on ion channels (Hille, 2001). Ion channels flux ions depending on either the presence of some neurotransmitter (ligand-gated channels, such as the AMPA channel), or the voltage across the channel (voltage-gated channels, such as the Na-channel). Ion channels are stochastic devices and are commonly described by Markov state diagrams. Typical states are the closed resting state and an open state, but many more intermediate states might be needed to accurately describe the kinetics. The actual transitions between states are stochastic, and the transition probabilities between the various states can depend on voltage or ligands. Ion channels are one of the main noise sources in the nervous system, and noise from channels can manifest itself in many ways, such as variations in spike timing or variability in the synaptic current.

In this study we consider stochastic synaptic channels, which are rapidly opened by some neurotransmitter. The fluctuations in the amplitude of the current through such a channel have been studied extensively. Typically there is one open state and thus the current is either maximal or zero. As a result the current through multiple independent parallel channels is distributed according to the binomial distribution. This observation forms the basis for noise analysis. Noise analysis extracts the unitary conductance of single channels from the noise in the current from an ensemble of channels.

As important as the current is the temporally integrated current, i.e. the total charge transported through the channel $Q=\int I(t) d t$. Its fluctuations have physiological importance. In an integrate-and-fire neuron a spike is generated whenever the voltage reaches a threshold. Hence if inputs are much faster than the leak time-constant, the spiking occurs when the net charge influx has reached a threshold. Here we calculate the mean charge and its fluctuations, first for a simple two-state model, and then for arbitrary state diagrams. We use these results to gain insight to the charge noise of synaptic channels, and to introduce a novel noise analysis technique based on charge.

\section{Fluctuations in single two-state channels}

\section{Fluctuations in currents}

We start with the simplest synaptic channel, a 2-state channel. We assume that at time 0 it is in the open state (due to the action of some transmitter), and subsequently closes at a random time with a rate constant $k=1 / \tau$,

$$
C \leftarrow O
$$

where $C$ denotes the closed state, and $O$ the open state. In the open state a current $i_{0}$ flows which we will call the unitary current of the channel, Fig. 1A shows the setup. Assuming Ohmic conductance the current is the product of the unitary conductance and the difference between membrane and synaptic potential, $i_{0}=g_{0}\left(V-V_{\text {syn }}\right)$. Throughout we will assume that the voltage difference is constant, for instance due to the presence of a voltage clamp, so that conductance and current are up to a factor identical. Note however, that for large synapses located far away from the clamping site, the synaptic current can substantially depolarize the 
A
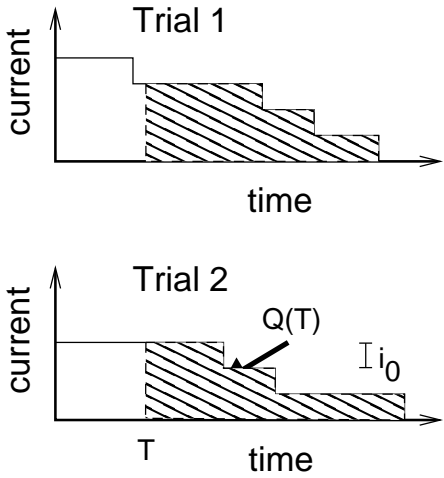
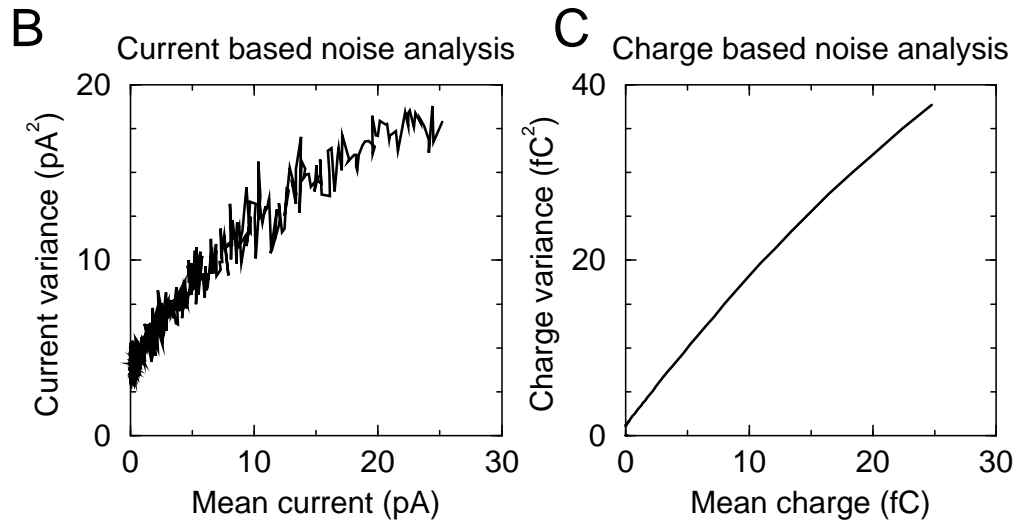

Figure 1: A). Schematic of the synaptic currents. After transient application of transmitter at time zero, a certain number of channels opens, each with a unitary current $i_{0}$. They close independently at a random time. The charge $Q(T)$ is defined as the integral of the current from time $T$ until all channels close (shaded area). $B+C$ ). Current based noise analysis (B) and charge based noise analysis $(C)$. When the variance of the current or charge is plotted against its mean, a parabola results, albeit with different parameters. Both analysis techniques lead to a reliable extraction of the number of channels in the synapse and the single channel properties (see text).

membrane so that this assumption no longer holds (the space-clamp problem) (Spruston et al., 1993).

At some random time the channel will close and it will not open again until transmitter is again released and binds to the channel. At any instance the channel will have a certain open probability $p(t)$, where $t$ denotes the time since the neurotransmitter pulse. When averaged over many trials, the average current is $\langle I(t)\rangle=i_{0} p(t)$, where the angular brackets denotes the average over multiple trials. Because the different trials are independent the variance is

$$
\begin{aligned}
\left\langle\delta I(t)^{2}\right\rangle & =\left\langle I(t)^{2}\right\rangle-\langle I(t)\rangle^{2} \\
& =i_{0}^{2} p(t)-\langle I(t)\rangle^{2} \\
& =i_{0}^{2} p(t)[1-p(t)] .
\end{aligned}
$$

where we denote the variance of a variable $x$ as $\left\langle\delta x^{2}\right\rangle$, defined as $\left\langle\delta x^{2}\right\rangle=\left\langle x^{2}\right\rangle-\langle x\rangle^{2}$.

When $N$ parallel, independent channels are present in the synapse, both the average and variance are $N$ times larger. Thus the mean is $\langle I(t)\rangle=N i_{0} p(t)$ and the variance $\left\langle\delta I(t)^{2}\right\rangle=$ $N i_{0}^{2} p(t)[1-p(t)]$, which equals the well-known binomial variance. Eliminating $p(t)$ yields,

$$
\left\langle\delta I(t)^{2}\right\rangle=i_{0}\langle I(t)\rangle-\frac{1}{N}\langle I(t)\rangle^{2} .
$$

The relation can be interpreted as follows: when at various time points the variance is plotted as a function of the mean current, a parabola results. A fit of a second order polynomial to this curve can thus extract the unitary current (from the linear term) and the number of channels (from the quadratic term). This is known as non-stationary noise analysis, and is valid for any kinetic scheme as long as there is only one open state (see appendix and Sigworth, 1980). An example is shown in Fig. 1B, further explained below. Noise analysis is a widely applied tool (Traynelis and Jaramillo, 1998). It can for instance be applied to determine if a change in synaptic strength is due to an increase in the number of channels, or a modulation of the single channel current, yielding insight in the underlying processes that caused the change. 


\section{Fluctuations in charge}

We now calculate the average charge and its fluctuations for this two-state channel. The time of the switch to the closed state is a random (Poisson) process, therefor the open-time of the channel has an exponential distribution $P\left(t_{\text {open }}\right)=\frac{1}{\tau} \exp \left(-t_{\text {open }} / \tau\right)$, where $\tau$ is the mean open time of the channel. The charge follows directly from the open-time as $Q=\int_{0}^{\infty} d t I(t)=$ $i_{0} t_{\text {open }}$. (For practical application the upper integration bound is taken so that all channels are very likely to have closed). Like the open time, the charge thus also has an exponential distribution, with a mean $q_{0}$ and a standard deviation that is equal to the mean, where we define the mean unitary charge as

$$
q_{0}=i_{0} \tau
$$

The mean unitary charge is the average charge that fluxes through a single channel following a single synaptic event. For $N$ parallel channels the total charge follows a gamma distribution of order $N$,

$$
P(Q)=\frac{1}{(N-1) !} \frac{1}{q_{0}}\left(\frac{Q}{q_{0}}\right)^{N-1} \exp \left(-Q / q_{0}\right) .
$$

This result can be easily understood. The gamma distribution describes the total waiting time of waiting for $N$ subsequent Poisson events. Due to the direct relation between charge and time, Fig. 1A, the total charge behaves as the total waiting time.

Next, we generalize two-fold. First we consider that the transmitter opens the single channel at time zero with only a limited probability $p(0)$. Secondly, we measure the charge as measured from an arbitrary time $T$ until all channels have closed, that is $Q(T)=\int_{T}^{\infty} I(t) d t$, see Fig. 1A. One finds for the mean of this quantity

$$
\langle Q(T)\rangle=p(T) q_{0}
$$

and for the variance

$$
\left\langle\delta Q^{2}(T)\right\rangle=q_{0}^{2} p(T)[2-p(T)] .
$$

It is of interest to compare this to the equation for the current noise. The term in the square brackets in Eq.4 is larger than in the corresponding equation for the current, Eq. 1. In other words, the charge in this kinetic scheme is for any open probability noisier than the current. This can be understood easiest in the case that at peak the channel is always opened by the transmitter, i.e. $p(0)=1$. In that case there will be no fluctuations at all in the peak amplitude $\left\langle\delta I^{2}\right\rangle=0$. In contrast, Eq.4 yields $\left\langle\delta Q^{2}(0)\right\rangle=q_{0}^{2}$, as the charge will still show fluctuations as the decay back to the rest-state occurs randomly. Equivalently, we can use the Coefficient of Variation to quantify the noise relative to the mean. The squared Coefficient of Variation of the charge is $C V_{Q}^{2}=2 / p(0)-1$, hence minimally $C V_{Q}=1$, but for the current only $C V_{I}^{2}=1 / p(0)-1$.

It is again possible to express the variance as a function of the mean, which for $N$ channels becomes

$$
\left\langle\delta Q^{2}(T)\right\rangle=2 q_{0}\langle Q(T)\rangle-\frac{1}{N}\langle Q(T)\rangle^{2}
$$

In analogy with the non-stationary current noise analysis, this relation can be used to extract the unitary $q_{0}$ and the number of channels from a set of records of synaptic currents. For this two-state channel the unitary current can be extracted as $i_{0}=q_{0} / \tau$. 


\section{Noise analysis based on charge}

The above result can be used to do noise analysis based on the noise in the charge. The procedure is to plot the variance versus the mean charge as the time $T$ is varied parametrically. The linear and quadratic coefficients of the polynomial fit will yield $q_{0}$ and $N$, respectively. This "charge-based" noise analysis is illustrated in Fig. 1C and compared to the current based noise analysis, Fig. 1B. For the figure we simulated 50 channels with $50 \%$ open probability at time zero, $1 \mathrm{pA}$ unitary current and $1 \mathrm{~ms}$ time constant, so that the unitary charge was $1 \mathrm{fC}$. A perfect clamp was assumed. White noise with a standard deviation of $2 \mathrm{pA}$ was added to mimic experimental noise (code online, see Appendix). Averages and variances of currents and charges were measured across 200 simulated events. To determine the accuracy and reliability of the method, we ran the simulation 50 times. The charge based analysis estimated $N=51 \pm 25$, $q_{0}=(1.03 \pm 0.10) \mathrm{fC}$, while the current based analysis yielded $N=55 \pm 14, i_{0}=(0.98 \pm 0.09) \mathrm{pA}$. So both methods are comparable, however, there are differences to point out:

1) In contrast with the current based analysis, the equation for the charge fluctuation is only valid for the simple kinetic scheme used here (see below for the generalization). The underlying reason is that the charge is determined not only by the instantaneous state of the channel but also by the future states. Thus the dynamics of the channel will matter.

2) The curve of the charge variance versus mean charge is much smoother than the curve for the current, compare Fig. 1B to Fig. 1C. The reason is that neighboring data points in the charge curve are highly correlated; $Q(t)$ and $Q(t+\delta t)$ share a large part of the time integral over the current. As a practical issue, most fitting algorithms assume independent noise and might over-estimate the confidence intervals of the fits. Fitting on subsets of the data as we did above will give reliable error bars.

3) Although the estimates for unitary current/charge are of comparable accuracy, the charge based analysis gave more variable results for the estimate of $N$. The reason is that estimating $N$ corresponds to estimating how strongly the parabola in Fig. 1C bends over, which is harder for the current than for the charge parabola. Estimating the unitary current/charge corresponds to estimating the slope near the origin, which is equally easy in both cases. In an effort to reduce the variability we tried fitting algorithms that correct for the correlation in the data (Heinemann and Conti, 1992; Steffan and Heinemann, 1997). However, neither a weighted least squares fit (assuming uncorrelated data with unequal variance), nor a general least squares fit (assuming correlations in the data), lead to marked improvement.

\section{Charge fluctuations for arbitrary channels}

In the appendix we derive the charge fluctuations for channels with an arbitrary state diagram. In case there is just one conducting state, it turns out that the variance in the charge behaves as

$$
\left\langle\delta Q^{2}(T)\right\rangle=\gamma\langle Q(T)\rangle-\frac{1}{N}\langle Q(T)\rangle^{2}
$$

The variance vs. mean relation has a quadratic form. The linear term, $\gamma$, has the dimension of charge, and we call it the charge noise constant. It is completely determined by the kinetics of the channel (see Appendix) and is independent of the initial state of the channel. For the simple two-state channel above one has $\gamma=q_{0}=i_{0} \tau$. For more complicated channels $\gamma$ is proportional to $i_{0}$, but knowledge of the state diagram is needed to recover $i_{0}$ exactly. 
Independently of the channel's kinetic scheme, the number of channels can be extracted from the quadratic term.

\section{Examples of charge fluctuations in various state diagrams}

As an illustration of the results above and in the Appendix, we analyze three simple state diagrams. For compactness we set $N=1$ and $i_{0}=1$. In the first state diagram there are two closed states: the resting state $C$, and an intermediate closed state $C^{\prime}$ which can have transitions back and forth to the open state. This channel will flicker before finally closing.

$$
C<C^{\prime} \rightleftharpoons O
$$

For this state diagram, the mean charge is $\langle Q(0)\rangle=\left[k_{C^{\prime} O} \pi_{2}(0)+\left(k_{C^{\prime} O}+k_{C^{\prime} C}\right) \pi_{3}(0)\right] / k_{O C^{\prime}} k_{C^{\prime} C}$, where $\pi_{2}(0)$ is the probability that the channel is in the $C^{\prime}$ state at time zero, and $k_{C^{\prime} O}$ denotes the rate for the transition from $C^{\prime}$ to $O$. The charge noise constant is $\gamma=2 \frac{k_{C^{\prime} O}+k_{C^{\prime} C}}{k_{C^{\prime} C} k_{O C^{\prime}}}$. The $C V^{2}=\frac{2\left(k_{C^{\prime} O}+k_{C^{\prime} C}\right)}{k_{C^{\prime} O} \pi_{2}(0)+\left(k_{C^{\prime} O}+k_{C^{\prime} C}\right) \pi_{3}(0)}-1$. It can be seen that $\min (C V)=1$, as in the two-state channel above. So the charge of the channel is equally noisy as the two-state channel.

In the second example the open state can decay to the resting state and a desensitized state, $C^{\prime}$.

$$
C<O \rightleftharpoons C^{\prime}
$$

For this case, the mean charge is $\langle Q(0)\rangle=\left[\pi_{2}(0)+\pi_{3}(0)\right] / k_{O C}$ and the charge noise constant is $\gamma=2 / k_{O C}$. The $C V^{2}=\frac{2}{\pi_{2}(0)+\pi_{3}(0)}-1$, which again is minimally one. Although we haven't proven this, these results suggest that a minimal $\mathrm{CV}$ of one for channels with one open state.

Finally, we consider a multi-step shutoff channel. There are two open states, with identical conductance

$$
C<O<O^{\prime}
$$

In addition we assume that both transitions are equal $k_{O C}=k_{O^{\prime} O}=k$. In analyzing this scheme one finds that the Markov matrix $M$ can not be diagonalized if both transitions are identical. We have ignored this case so far, as its treatment is standard, but somewhat tedious (Greenberg, 1998). One can simply set the transition rates arbitrarily only to equate them in the final formulae.

In this case the charge is the sum of an exponential and an alpha-function $\langle Q(T)\rangle=$ $\frac{1}{k}(2+k T) e^{-k T}$, while $\left\langle Q^{2}(T)\right\rangle=\frac{6+2 k T}{2 k+k^{2} T} i_{0}\langle Q(T)\rangle$. Importantly, the relation between $\left\langle Q^{2}(T)\right\rangle$ and $\langle Q(T)\rangle$ now depends on time (Eq. 10 in the Appendix is violated). As a result the meanvariance plot in noise analysis will not yield a parabola. This due to the fact that there are two open states.

Interestingly, the $C V^{2}=\frac{2 \pi_{2}(0)+6 \pi_{3}(0)}{\left[\pi_{2}(0)+2 \pi_{3}(0)\right]^{2}}-1$, which reaches a minimum of $1 / 2$ for $\pi_{2}(0)=0$, $\pi_{3}(0)=1$. Hence the multi-step shut-off reduces the variability in the charge. For $n$ open states with identical rates, the charge will have a gamma-distribution with $C V^{2}=1 / n$. A very similar mechanism has been hypothesized to reduce response variations in the photo-receptor (Field and Rieke, 2002). 
A
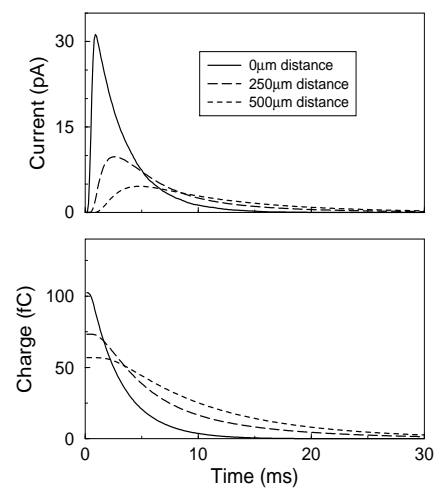

B
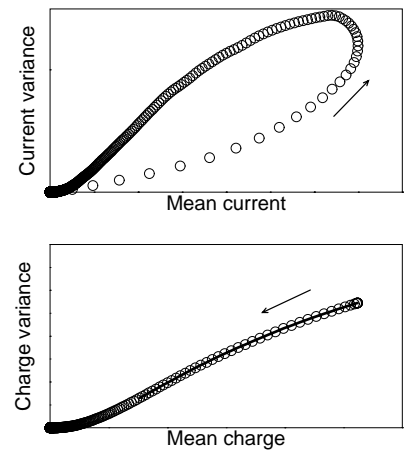

$\mathrm{C}$
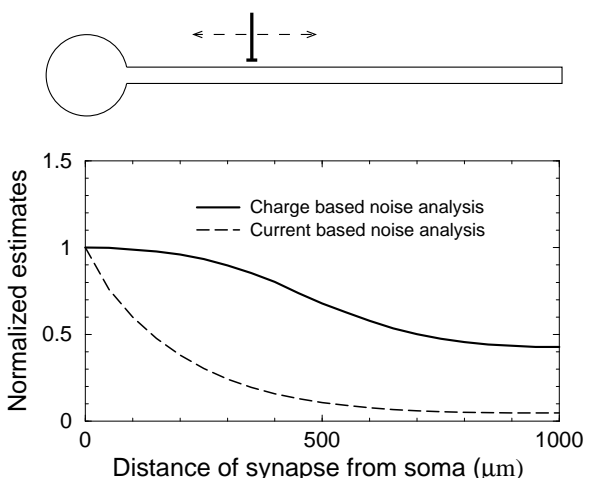

Figure 2: Current and charge fluctuations in a simulated dendrite with a single synapse at a varying location. A) The average synaptic currents (top) and charge, $\int_{T}^{\infty} I(t) d t$ (bottom) for a synapse located 0,250 and $500 \mu \mathrm{m}$ from the voltage clamped soma. B) The variance versus mean relation of current (top) and charge (bottom) for a synapse located $250 \mu \mathrm{m}$ from the soma. The cable filtering distorts the parabolas (compare to Fig. 1). The arrow denotes increasing time. The solid line in the bottom panel is the quadratic fit to the restricted data. C) Normalized estimates of the unitary current and charge noise constant as a function of distance from the soma. The charge-based analysis is less biased by cable filtering.

\section{Noise analysis of cable filtered events}

We now return to channels with one open state and the application to noise analysis. The formulation of the noise in the charge rather than the noise in the currents has advantages when performing noise analysis for synapses on the dendrite far away from the clamping site. The rapidly changing synaptic currents are strongly filtered by the cable, but the charge is filtered much less. This is shown in Fig. 2A where current and charge are plotted for synapses located at different positions along a dendrite. The peak current is much more attenuated by the cable filtering than the charge. The underlying reason is that the electrotonic length is frequency dependent; high frequency signals, including transients are filtered more strongly than DC components (e.g. Koch, 1999).

We simulated a simplified setup with one dendritic passive cable connected to a soma, using the NeuronC simulator (Smith, 1992). Although this is a simplified model, it is not unrealistic as under certain conditions dendritic trees can be collapsed into a single equivalent cable (e.g. Koch, 1999). The soma had a diameter of $10 \mu \mathrm{m}$, the dendrite had a diameter of $1 \mu \mathrm{m}$ and a length of $1000 \mu \mathrm{m}$. The membrane resistance was $20 \mathrm{k} \Omega / \mathrm{cm}^{2}$ and the internal resistance was $200 \Omega \mathrm{cm}$ (electrotonic length at zero frequency was $707 \mu \mathrm{m}$ ). An AMPA type synapse was positioned at varying locations along the dendrite. The AMPA synapse was modeled according to the 7-state diagram with a single open state provided in (Jonas et al., 1993), but de-sensitization was turned off to ensure stationarity (for details see AMPA type 5 in the NeuronC documentation). The synapse contained 50 channels with a conductance of $20 \mathrm{pS}$ each. The peak open probability was about $50 \%$. At every synapse location 200 synaptic events were simulated, while the soma was clamped at $-70 \mathrm{mV}$. As the synaptic currents were rather small, the local voltage excursion was maximally $10 \mathrm{mV}$ above the clamping voltage.

As can be seen in Fig 2B, both the current (top) and charge (bottom) variance vs mean curves are distorted due to the cable filtering. For the charge, a supra-linear relation between variance and mean appears near the origin (corresponding to long times $T$ ). This will lead to 
A
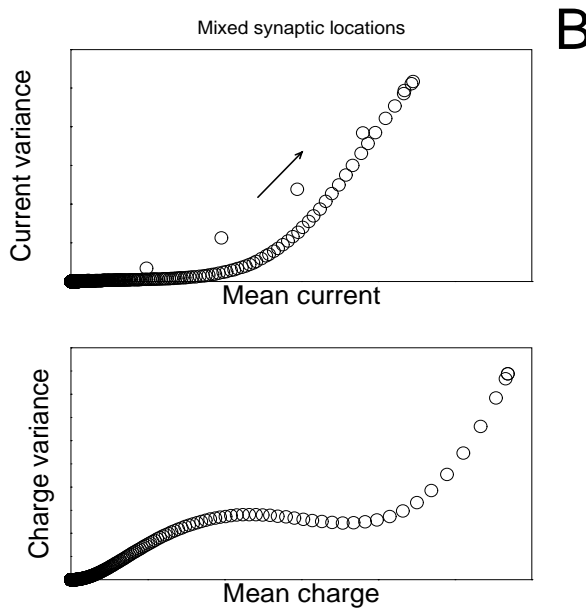
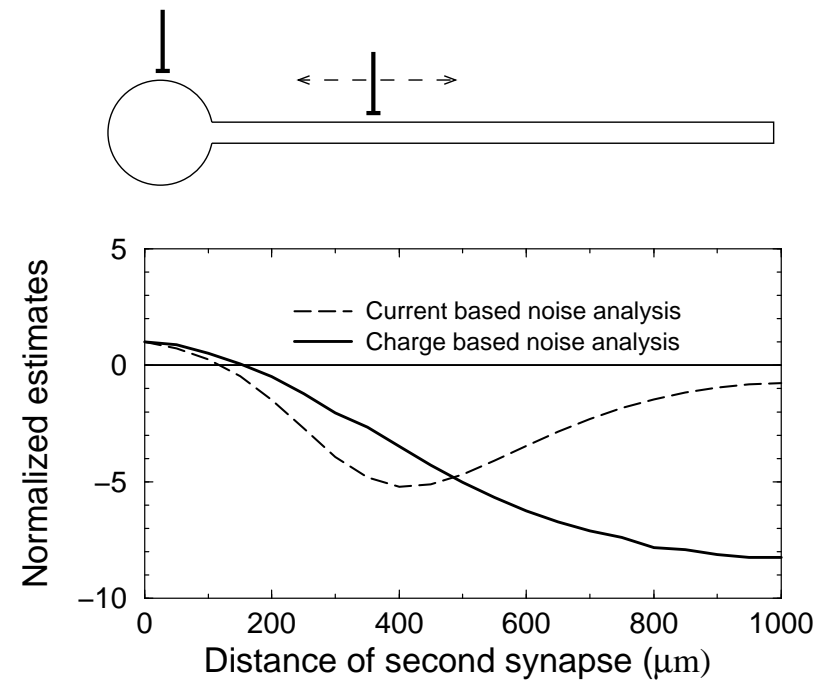

Figure 3: Break down of noise analysis when multiple synapses are distributed along dendrite. The synapses are asynchronously activated. A) Variance versus mean plots in one synapse is at the soma and the other synapse is located $500 \mu \mathrm{m}$ away on the dendrite. Top graph: current, bottom graph: charge B) Normalized estimates of the unitary current and charge noise constant versus the location of the second synapse, the first synapse being at the soma. Only when the two synapses are close the estimate is reasonable, otherwise even negative estimates can result.

a poor fit to the parabola. However, the concave parabola is still apparent for larger mean currents. An ad hoc cut-off restricting the fit above $30 \%$ of the maximal charge leads to a good fit (solid line in bottom panel).

The results of the both noise analysis techniques are shown in Fig. 2C. For ease of comparison both are plotted relative to the estimate obtained when the synapse is located at the soma. The unitary current is strongly underestimated away from the soma, because the current noise is strongly filtered and the reduced noise is interpreted as stemming from many, small conductance channels. In contrast the estimated charge noise constant is much less affected by the filtering. Note, that both estimates flatten out at the end of the cable. This is due to the sealed end at the end of the dendrite.

\section{Pooling from synapses with different locations}

Finally we consider a situation with two synapses located at different positions on the cell. The synapses are assumed to be independently active so that compound events are negligible. The charge based as well as the traditional current based analyses break down in this case. This is illustrated in Fig.3, where one synapse was located at the soma and the location of the other synapse was on the dendrite. Both variance versus mean plots in Fig.3A deviate considerably from the ideal downwards curved parabola (cf. Fig.1). In Fig.3B, the location of the second synapse was varied systematically, and the channel properties were estimated. Only when the second synapse is close to the first one, the estimates are reasonable (Benke et al., 2001). If the synapses are located further apart, negative estimates result, which are clearly non-sense.

The reason for the failure is that both methods confuse the variations among the events caused by differential filtering, for fluctuations caused by stochastic channels. In this highly 
idealized case it might still be possible to separate the events based on their shape and carry out the analysis on for each synapse individually. When recording from multiple synapses distributed over the tree, that would be likely impossible.

\section{Discussion}

We have considered the open state of synaptic channels and derived expressions for its mean open time and its fluctuations. For channels with a single open state, the variance of the charge fluctuation is a quadratic function of the mean charge, characterized by the charge noise constant $\gamma$. The result has a number of applications: First, it poses fundamental constraints on the noise in neurons, as it calculates the charge noise given the channel's state diagram. One can wonder if neural firing is more affected by current noise or by charge noise. There is no easy answer to this question, and the two noise sources are related. For instance, if the input is much faster than the membrane time-constant, the membrane voltage is determined by the charge influx, and the neuron has a charge threshold. But for a very slowly varying input, the integrate-and-fire neuron has a current threshold. For the Hodgkin-Huxley model the situation is even more complicated and follows an intermediate behavior, for a discussion see (Koch, 1999). However, it should also be noted that simulations suggest that noise stemming from the stochastic nature of synaptic channels is negligibly small, compared to for instance probabilistic vesicle release which affects all channels in the synapse collectively (van Rossum et al., 2003).

Secondly, the results can be used to extract fundamental synaptic parameters in a way that is relatively insensitive to cable filtering. It thereby overcomes some of the problems with current based noise analysis, which is applied mostly to inhibitory synapses believed to be close to the soma (e.g. Kilman et al., 2002). We note that current based noise analysis has been further developed: Peak-scaled noise analysis of synaptic currents can be used to estimate unitary currents when pooling from a population of synapses with different $N$ (Traynelis et al., 1993; De Koninck and Mody, 1994). It is not obvious how the charge based noise analysis is to be extended for this purpose.

Importantly, when synaptic events stemming from different parts of the dendrite are pooled, any noise analysis is likely to fail, because it will confuse deviations from the mean due to noise with deviations due to the differential filtering. We find that both the current and the charge method break down. This means that the method is best applied to situations in which always the same synapse is stimulated, e.g. using glutamate uncaging.

We note that in principle in our formalism it should be possible to calculate the exact effect of cable filtering on both current and charge fluctuations. This is outside of the scope of this paper, but might eventually provide even better results and remove the reliance on the somewhat arbitrary restriction in the charge fit.

Finally we note that our results have a much wider application beyond synaptic channels. Any Markov process that is triggered by an external event and then decays back to the resting state falls under this formalism. For instance, the transduction cascade in the photo-receptors after absorption of a photon can be described this way, and analysis of the fluctuations have been used to infer properties of the underlying transduction cascade (Field and Rieke, 2002). Similarly, one can consider the activation of stochastic plasticity networks that are triggered by brief Ca influxes (Hayer and Bhalla, 2005). 


\section{Acknowledgments:}

HFD was supported by the German Federal Ministry of Education and Research (grant 01GQ0420). ABB was supported by the HFSP.

\section{Appendix: Charge fluctuations for arbitrary channels}

Here we derive a general expression for the charge fluctuations, assuming that the channel dynamics are described by a Markov process. The evolution of the states of the channel obeys $\frac{d \boldsymbol{\pi}(t)}{d t}=M \boldsymbol{\pi}(t)$, where $M$ is the Markov matrix (zero column sum), which is constructed from the transition rates between states $c_{i j}$ as $M_{i j}=c_{i j}-\delta_{i j} \sum_{k} c_{k j}$. For reviews with example state diagrams, see e.g. Destexhe et al. (1998); Roth and van Rossum (2009). The probability $\boldsymbol{\pi}(t)$ is the occupancy probability for each state, written as vector (we use boldface to denote vectors and capital letters from matrices). Of particular relevance are the distribution right after transmitter application, $\boldsymbol{\pi}(t=0)$ and the equilibrium distribution $\boldsymbol{\pi}(t=\infty)$.

Each state has a certain current associated with it. This is denoted by the vector $\mathbf{s}$, so that the mean current through a single channel at any instant is given by $i(t)=\mathbf{s} . \boldsymbol{\pi}(t)$, where the dot stands for the dot product. Typically only one of the states will be an open state. So for a 3 -state channel with only the $3 r d$ state conducting, $\mathbf{s}$ takes the form $\mathbf{s}=\left(0,0, i_{0}\right)$ and $i(t)=i_{0} \pi_{3}(t)$. However, the formalism presented here allows for state-diagrams in which there are multiple open states, potentially with different conductances.

\section{Mean current and charge}

We first calculate the mean current and charge through a single channel. The time evolution of the states is best described in the eigenspace of the transition matrix $M$. The matrix $M$ is diagonalized as $V^{-1} M V=\operatorname{diag}\left(\lambda_{1}, \lambda_{2}, \ldots \lambda_{N}\right)$. The matrix $V$ transforms from eigenvector space to regular space; each column is an eigenvector of the $M$ matrix ${ }^{3}$. The Perron-Frobenius theorems warrant that there is one zero eigenvalue, and the others are negative, although they can be complex (e.g. van Kampen, 1992). Each eigenvector decays exponentially according to its eigenvalue $\lambda_{i}$. The trick is to transform to the eigenspace, evolve the dynamics, and transform back. That means that the occupancy at time $t$ obeys $\boldsymbol{\pi}(t)=V D\left(e^{\boldsymbol{\lambda} t}\right) V^{-1} \boldsymbol{\pi}(0)$, where $D\left(e^{\boldsymbol{\lambda} t}\right)=\operatorname{diag}\left(e^{\lambda_{1} t}, e^{\lambda_{2} t}, \ldots\right)$ denotes a diagonal matrix with the exponentiated eigenvalues $e^{\lambda_{i} t}$ on the diagonal. From this the mean current at time $t$ follows

$$
\begin{aligned}
\langle I(t)\rangle & =\mathbf{s} \cdot \boldsymbol{\pi}(t) \\
& =\mathbf{s} \cdot V D\left(e^{\boldsymbol{\lambda} t}\right) V^{-1} \boldsymbol{\pi}(0) .
\end{aligned}
$$

As one eigenvalue is zero, this means that the current is in general the sum of $(S-1)$ exponentials, where $S$ is the number of states. The mean charge follows as

$$
\begin{aligned}
\langle Q(T)\rangle & =\int_{T}^{\infty}\langle I(t)\rangle d t \\
& =-\mathbf{s} \cdot V D\left(\frac{e^{\boldsymbol{\lambda} T}}{\boldsymbol{\lambda}}\right) V^{-1} \boldsymbol{\pi}(0) .
\end{aligned}
$$

\footnotetext{
${ }^{3}$ In matlab it is the result of the command $[V, \lambda]=e i g(M)$, while Mathematica's 'EigenSystem $]$ ' command returns $V^{T}$.
} 
where $D\left(\frac{e^{\lambda T}}{\lambda}\right)=\operatorname{diag}\left(e^{\lambda_{1} T} / \lambda_{1}, e^{\lambda_{2} T} / \lambda_{2}, \ldots\right)$.

An acute reader might have noticed that because the matrix $M$ always has a zero eigenvalue, this expression might diverge. However, as long as the equilibrium state $\boldsymbol{\pi}(\infty)$ does not conduct, this divergence does not show up in the outcome of Eq. 7. For the numerical calculation one can replace the zero eigenvalue by an arbitrary number; its value should not matter.

\section{Variance in current and charge}

The variance of the charge can be written as $\left\langle Q^{2}(T)\right\rangle=\left\langle\int_{T}^{\infty} d t \int_{T}^{\infty} d t^{\prime} I(t) I\left(t^{\prime}\right)\right\rangle$. Thus we need to know $\left\langle I(t) I\left(t^{\prime}\right)\right\rangle$, which is again calculated by using the evolution in eigenspace. Assuming $t^{\prime}>t$,

$$
\begin{aligned}
\left\langle I(t) I\left(t^{\prime}\right)\right\rangle & =\sum_{i j} s_{j} P\left(j, t^{\prime} \mid i, t\right) s_{i} \pi_{i}(t) \\
& =\mathbf{s} \cdot V D\left(e^{\boldsymbol{\lambda}\left(t^{\prime}-t\right)}\right) V^{-1} D(\mathbf{s}) \boldsymbol{\pi}(t) \\
& =\mathbf{s} \cdot V D\left(e^{\boldsymbol{\lambda}\left(t^{\prime}-t\right)}\right) V^{-1} D(\mathbf{s}) V D\left(e^{\boldsymbol{\lambda} t}\right) V^{-1} \boldsymbol{\pi}(0),
\end{aligned}
$$

where $D(\mathbf{s})=\operatorname{diag}\left(s_{0}, s_{1}, \ldots\right)$. Note that the time-dependent variance of the current follows from this expression as

$$
\left\langle\delta I(t)^{2}\right\rangle=\mathbf{s} D(\mathbf{s}) \cdot \boldsymbol{\pi}(t)-[\mathbf{s} . \boldsymbol{\pi}(t)]^{2} .
$$

If there is just one open state $\mathbf{s}=\left(0,0, \ldots, i_{0}, \ldots 0\right)$ with an associated probability $p(t)$, we retrieve the well-known binomial variance $\left\langle\delta I(t)^{2}\right\rangle=i_{0}^{2} p(t)[1-p(t)]$ of a single channel derived above.

Continuing with the calculation of the variance of the charge, the second moment of the charge is given by

$$
\begin{aligned}
\left\langle Q^{2}(T)\right\rangle & =2 \int_{T}^{\infty} d t \int_{t}^{\infty} d t^{\prime}\left\langle I(t) I\left(t^{\prime}\right)\right\rangle \\
& =2 \mathbf{s} \cdot V D(1 / \boldsymbol{\lambda}) V^{-1} D(\mathbf{s}) V D\left(e^{\boldsymbol{\lambda} T} / \boldsymbol{\lambda}\right) V^{-1} \boldsymbol{\pi}(0),
\end{aligned}
$$

from which the variance follows as $\left\langle\delta Q^{2}(T)\right\rangle=\left\langle Q^{2}(T)\right\rangle-\langle Q(T)\rangle^{2}$, and for $N$ channels $\left\langle\delta Q^{2}(T)\right\rangle=\left\langle Q^{2}(T)\right\rangle-\frac{1}{N}\langle Q(T)\rangle^{2}$. Eq. (9) is the main result of this section. Analytic results for channels with 4 or more states becomes quickly unwieldy, however despite the complicated appearance, numerical evaluation is straightforward.

Channels with a single open state

If there is one open state, the calculation simplifies considerably. Suppose that only state $m$ conducts, $s_{i}=i_{0} \delta_{i, m}$. Now from Eq.(9),

$$
\left\langle Q^{2}(T)\right\rangle=\gamma\langle Q(T)\rangle,
$$

with the constant $\gamma=2 i_{0}\left[V D(1 / \boldsymbol{\lambda}) V^{-1}\right]_{m m}$, where $[A]_{m m}$ indicates the matrix element $A_{m, m}$.

The constant $\gamma$ is completely determined by the kinetics of the channel and is independent of the initial state of the channel, i.e. the value of $\boldsymbol{\pi}(0)$. Furthermore, because this constant is also independent of time, the variance vs. mean relation will have the quadratic form. The $\gamma$ has the dimension of charge, and we call it the charge noise constant. For the simple two-state 
channel above one has $\gamma=q_{0}$. However, in general, the charge noise constant is not equal to $q_{0}$. This is where the charge noise differs from current noise; for currents the linear term in the parabola, Eq.(2), equals the unitary current independent of kinetics.

For $N$ channels we find

$$
\left\langle\delta Q^{2}(T)\right\rangle=\gamma\langle Q(T)\rangle-\frac{1}{N}\langle Q(T)\rangle^{2} .
$$

Independent of the channel's kinetic scheme, the number of channels can be extracted from the quadratic term.

If more than one state is conducting the simplification does not hold and variance-mean data will in generally not fall on a parabola. Note that in that case, also the current based noise analysis Eq. (2) breaks down, and instead Eq.(8) should be used.

\section{Computer code}

The computer code for both the simulation and the analysis is available on homepages . inf.ed.ac.uk/mvanross/code.

\section{References}

Benke, T.A., Lüthi, A., Palmer, M.J., Wikström, M.A., Anderson, W.W., Isaac, J.T., Collingridge, G.L.. Mathematical modelling of non-stationary fluctuation analysis for studying channel properties of synaptic ampa receptors. J Physiol 2001;537(Pt 2):407-420.

De Koninck, Y., Mody, I.. Noise analysis of miniature IPSCs in adult rat brain slices: properties and modulation of synaptic GABAa receptor channels. J Neurophysiol 1994;71:13181335 .

Destexhe, A., Mainen, Z.F., Sejnowski, T.J.. Kinetic models of synaptic transmission. In: Koch, , Segev, , editors. Methods in neuronal modeling (2nd ed.). Cambridge: MIT Press; 1998 .

Field, G.D., Rieke, F.. Mechanisms regulating variability of the single photon response of mammalian rod photoreceptors. Neuron 2002;35:733-747.

Greenberg, M.D.. Advanced Engineering Mathematics. New York: Prentice Hall, 1998.

Hayer, A., Bhalla, U.S.. Molecular switches at the synapse emerge from receptor and kinase traffic. PLoS Comput Biol 2005;1(2):137-154.

Heinemann, S.H., Conti, F.. Nonstationary noise analysis and application to patch clamp recordings. Methods Enzymol 1992;207:131-148.

Hille, B.. Ionic Channels of excitable membranes. Sunderland, MA: Sinauer, 2001.

Jonas, P., Major, G., Sakmann, B.. Quantal components of unitary EPSCs at the mossy fibre synapse on CA3 pyramidal cells of rat hippocampus. J Physiol 1993;472:615-663.

Kilman, V., van Rossum, M.C.W., Turrigiano, G.G.. Activity Deprivation Reduces mIPSC Amplitude by Decreasing the Number of Postsynaptic GABAa Receptors Clustered at Neocortical Synapses. J Neurosci 2002;22:1328-1337. 
Koch, C.. Biophysics of computation. New York: Oxford University Press, 1999.

van Rossum, M.C.W., O'Brien, B.J., Smith, R.G.. The effects of noise on the timing precision of retinal ganglion cells. J Neurophysiol 2003;89:2406-2419.

Roth, A., van Rossum, M.. Modeling synapses. In: Schutter, E.D., editor. Computational Modeling Methods for Neuroscientists. MIT Press; 2009. .

Sigworth, F.J.. The variance of sodium current fluctuations at the node of Ranvier. J Physiol 1980;307:97-129.

Smith, R.G.. NeuronC: a computational language for investigating functional architecture of neural circuits. Journal of Neuroscience Methods 1992;43:83-108.

Spruston, N., Jaffe, D.B., Williams, S.H., Johnston, D.. Voltage- and space-clamp errors associated with the measurement of electrotonically remote synaptic events. J Neurophysiol 1993;70:781-802.

Steffan, R., Heinemann, S.H.. Error estimates for results of nonstationary noise analysis derived with linear least squares methods. J Neurosci Methods 1997;78(1-2):51-63.

Traynelis, S.F., Jaramillo, F.. Getting the most out of noise in the central nervous system. Trends in Neurosci 1998;21:137-145.

Traynelis, S.F., Silver, R.A., Cull-Candy, S.G.. Estimated conductance of glutamate receptor channels activated during EPSCs at the cerebellar mossy fiber-granule cell synapse. Neuron 1993;11:279-289.

van Kampen, N.G.. Stochastic processes in physics and chemistry. 2nd ed. Amsterdam: North-Holland, 1992. 